\title{
A Discriminative Color-to-Grayscale Representation for Retinal Vessel Segmentation
}

\author{
M Khan*, M Moatamedi, B Alzahabi \\ Al Ghurair University, Dubai Academic City, UAE
}

\begin{abstract}
Structural changes in retinal blood vessels are important indicators for many ocular diseases including diabetic retinopathy (DR), glaucoma, and hypertension. An automated vessel segmentation process almost always begins with acquired color fundus image, containing three layers of images (red, green and blue), and then quickly converted them to a single grayscale image. However, grayscale conversion is not unique, and more than one grayscale representation can be obtained for a given color image. For the many currently existing automated vessel extraction methods, the green channel of the RGB color fundus image is routinely used as an input greyscale representation to a pipeline of the segmentation process for the reason that it provides the best contrast among all three channels, namely red, green and blue. We hypothesize that vessel information contained in dropped channels, red and blue, will add to result in improved segmentation performance. In this paper, we propose a linear combination framework to utilize all three channels of the color fundus image based on their importance level rather than completely discarding some channels. We devised a Principal Component Analysis (PCA) method that provides appropriate weights for each color channel to realize a more discriminative grey-scale representation. The added information made available through PCA-based grayscale representation results in improved performance for automated vessel segmentation algorithms. The performance of the framework is analyzed on two publicly available databases (DRIVE, STARE) of fundus images quantifying improvements in all three aspects called accuracy, sensitivity, and specificity.
\end{abstract}

\section{INTRODUCTION}

Structural changes in retinal blood vessel network is a good indicator of the presence of ophthalmic diseases, e.g., hypertension, cardiovascular diseases, diabetic retinopathy, glaucoma, etc. $[1,2]$. Vessel segmentation of fundus images has played a major role in calibrating change that helps measure the severity of ocular diseases. However, periodic screening of retinal images is necessary to identify changes in vascular structure at an early stage that would be crucial in stopping major vision loss [2]. Therefore, to develop an accurate computerized diagnosis system for eye examination retinal vessel segmentation emerges as the prerequisite task. Extraction of retinal vessels in face of an uneven illuminated and 
pigmented fundus image becomes a challenging problem. Besides retinal blood vessels, the existence of other structures (e.g., exudates, optic disc, fovea, red lesions) makes the vessel detection even more elusive. Also, the vessel thickness in a retina varies in a wide range whereas thin vessels have low contrast which makes it harder for a computerized method to detect them [2].

Several automatic vessel segmentation of retinal fundus images have been proposed in literature. Fraz et al. have reported a comprehensive literature survey on retinal blood vessel segmentation in [3]. The vessel segmentation techniques can be broadly classified as: (i) supervised and (ii) unsupervised techniques. In supervised category, k-NN-classifier, Artificial Neural Network (ANN), trainable COSFIER filters, Support Vector Machine (SVM), Extreme Learning Machine (ELM), Deep Neural Network (DNN), etc. have been tested. However, supervised algorithms rely on robust feature extraction followed by an appropriate classifier. Since these approaches entail training process and thus become timeconsuming. In many approaches computationally, simple unsupervised methods such as line detector, Gabor filters, and gray level co-occurrence matrix (GLCM) based methodologies have been explored for feature extraction purpose. Among them the multi-scale line filtering as proposed in [4] has proven to be more effective in detecting thin low-contrast vessels. In these approaches, classification of the vessel or non-vessel pixels is decided without training process, i.e., training data do not contribute to finding the model parameter.

Though there is a subtle difference between methods belonging to two categories, there is an agreement in the general methodology for the purpose of vessel segmentation. The general process consists of three phases: First to convert the color fundus image to grayscale, second perform suitable geometrical filtering to boost vessels and to suppress the non-vessel objects, and third apply a threshold strategy to bifurcate the image into vessel and non-vessel regions. Considerable attention has been focused in the past on developing efficient filters for vessels and better threshold schemes. However, the first phase of color conversion remained largely unattended. Here we propose a novel technique of converting color fundus image to a grayscale representative that is more discriminative to the final task of separating vessels in an image.

Since the color-to-gray image conversion is basically a dimensionality reduction process, it results in some loss of information. Therefore, we should concentrate on finding an appropriate mapping method during the conversion from three-dimensional color images into one-dimensional gray images so as to minimize the loss of relevant information. Many recent studies have aimed to produce the perceptually discriminable gray images by using applying mapping methods. Grundland et al. [5] propose the Decolorize algorithm based on contrast enhancement of a converted gray image. By involving an image sampling and a subsequent dimensionality reduction process. Gooch et al. [6] present a neighborhood mapping algorithm called Color2Gray, which iteratively decides the gray levels that preserve better the color differences between all pairs of pixels. Smith et al. [7] present a mixture a local mapping method having global constraints to favor the perceptual accuracy rather than exaggerate the discriminability.

Due to the fact that dimension is reduced in a color-to-gray conversion process, Principal Component Analysis (PCA) [8] can be invoked that is known as a well-known standard linear technique to reduce dimensions of data by projecting the data onto orthogonal subspaces resulting in maximal data spread. Nevertheless, it is hard to separate samples in subspaces if the samples are clustered along projection directions in the original space. Therefore, an 
appropriate color space has to be searched that provide most of the relevant data spread along one dimension. We propose here a methodology that seeks to find first an appropriate color space and then use PCA-based color-to-gray conversion to solve this issue. The PCA-based color-to-gray image conversion method is supposed to effectively preserves and, in some cases, increase the discriminability between vessel and non-vessel classes in color image by simple linear computations in appropriate subspaces. As a result, we can retain the best possible gray-scale representation having high classification value with low computational complexity.

\section{METHOD}

The proposed method consists of a sequence of cascaded processes. The processing taking place at each stage is described as follows.

\subsection{Non-uniform lllumination Correction}

Fundus Images are captured in poor lighting conditions. In order to compensate the nonuniform illumination of retinal images the illumination distribution has to be estimated properly. However, this is not straightforward since the retina has several elements like the blood vessels or the optic disc which have different luminosity properties. Furthermore, the curved retinal surface and the geometrical configuration of the light source and camera lead to a poorly illuminated peripheral part of the retina with respect to the central region. The image thus possesses low dynamic ranges with high level of noise that is detrimental to the overall performance of vessel extraction algorithms. To make extraction algorithms robust in face of non-uniform conditions, then illumination correction has to be implemented to improve the contrast of vessels in an image. For this purpose, the input color fundus image is first inverted such whereas the background illumination variation appears as fog. The histogram of pixel-wise inversion of fundus images is found to be very similar to the histogram of hazy images. Therefore, the haze removal techniques can be utilized to make fundus images uniformly illuminated. The dehazed image as shown in Fig. 1(b) contains less illumination variation as most of the dark shadows suppressed around the periphery.

However, the dehazed fundus image do contain illumination variation around the optical disc region. In an effort to minimize this Optic disk illumination effect, this research team in [9] proposes a modified homomorphic filtering protocol that have been dubbed as Halo Compensated Homomorphic Unsharp Masking (HC-HUM). The protocol as a block diagram is depicted in Fig. 2. The HC-HUM approach is an incremental improvement upon the conventional homomorphic filter since it removes over-illumination artifacts from areas corresponding to the boundary regions of the larger blood vessels in a vascular network as well as suppress the optical disk over exposure. In order to achieve the objective of eliminating effects, the HC-HUM performs a gray level correction on the highest portion of a selected image's dynamics by effecting a non-linear gray level mapping followed by a dynamic recovery process. The HC-HUM process is implemented on all the three channels of the dehazed color image. 

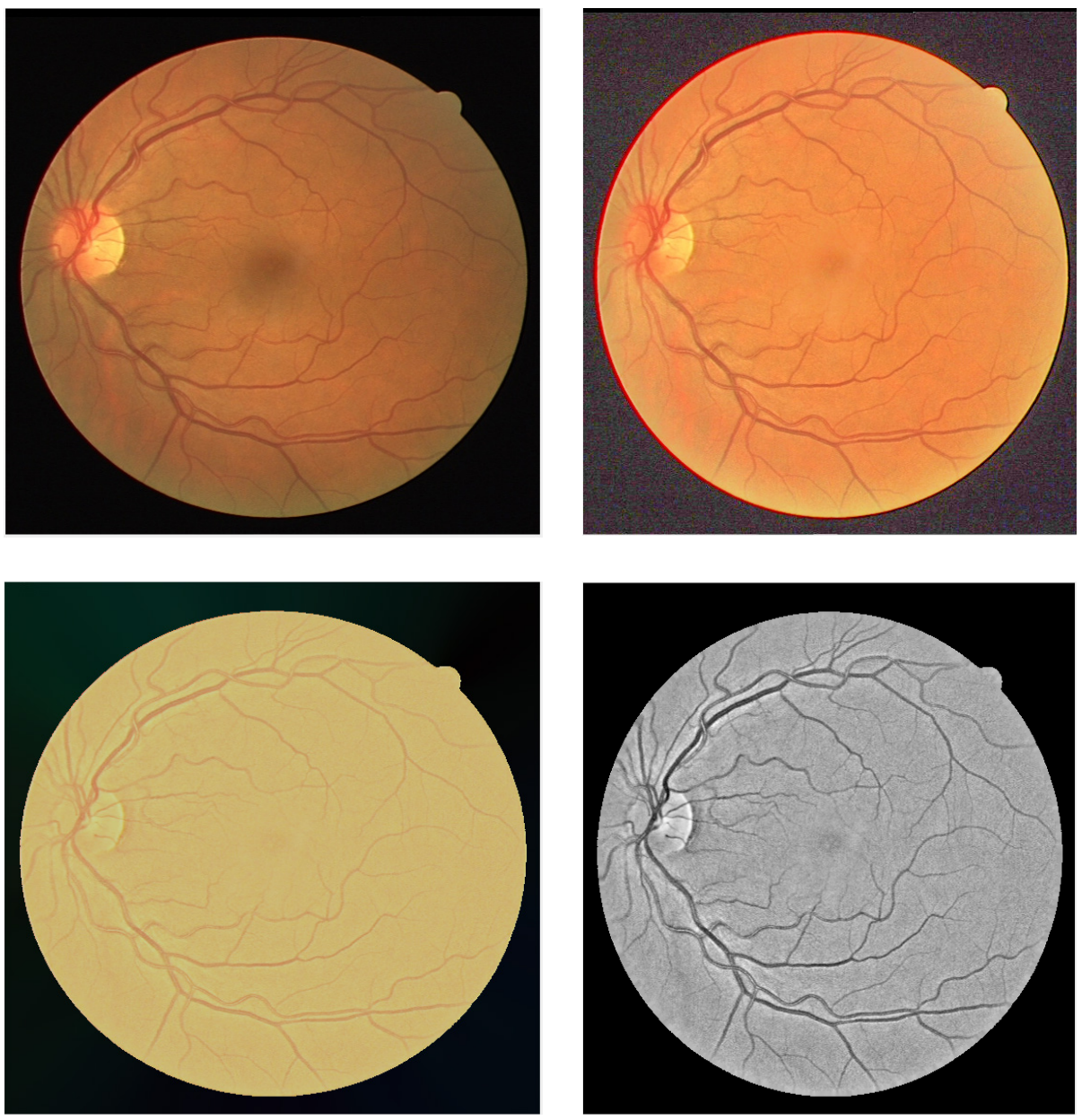

Figure 1. The first row displays on the left the acquired fundus image and on the right the dehazed image. While the second row depicts HC-HUM process output on the left and the PCA-based grayscale image on the right.

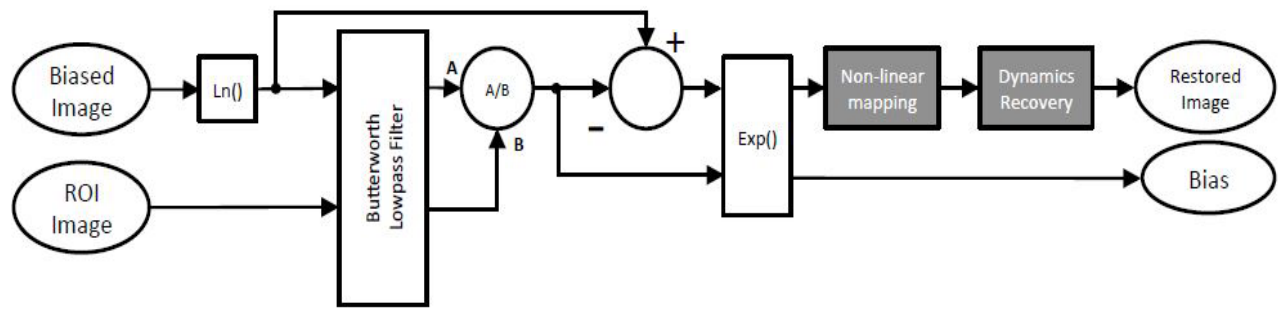

Figure 2. A complete pipeline of the Halo-Compensated Homomorphic Filtering Process. 


\subsection{PCA-based Color-to-Gray Conversion}

Now that most of the non-uniform illumination has been compensated and the resulting image contains largely variation in intensity due to vessels, we can move to the next stage of colorto-gray-scale conversion. Given that it is possible to produce more than one gray-scale representation for the same color image, it is important that we find a color-to-grayscale conversion method that results in optimum contrast between the vascular networks and the image background. Instead of adhering to the normal convention of using only green channel color data to generate gray-scale representations, we opted for employing principal component analysis (PCA) to achieve gray-scale conversion, as per the recommendation of [39]. Firstly, a vectored color image $\left(f_{r g b} \in \Re^{3}\right)$ is created by stacking image representations from the RGB color channels (i.e., red, green and blue). Subsequently, a zero-mean $\mathrm{YCbCr}$ image $\left(f_{y c c} \in \mathfrak{R}^{3}\right)$ is computed to separate the luminance and chrominance channels using the conventional transfer function $f($.$) . Finally, three eigenvalues \left(\lambda_{1} \geq \lambda_{2} \geq \lambda_{3} \in \Re^{3}\right)$, and their normalized eigen vectors $\left(v_{1}, v_{1}, v_{1} \in \mathfrak{R}^{2}\right)$ are computed via PCA. The resultant gray-scale image $\left(f_{\text {gray }} \in \mathfrak{R}^{2}\right)$ is by the weighted linear combinations of three projections as per their eigen values. The resultant image data obtained is mapped to the range $(0-255)$. It must be emphasized the derived eigenvalues are used as weighting factors for projection results on corresponding eigenvectors. As a result, the color-to-gray mapping is dominated by the first subspace projection, and the second and third subspace projections contribute to preserving details of a color image in a gray image. Fig. 3 the visualizes overall conversion process for an input color image by exemplifying resulting images. The histogram of fgray in shown on left side of Fig. 3 contains a wider range of data points compared to that of fg green image, which signifies that this particular color-to-grayscale conversion technique yields more spread of data than methods based on green channel conversion.

\subsection{Multi-Scale Line Filtering}

The retinal vasculature appears as piecewise linear features having variation in width and many branches sprouting within the retinal image. The basic line detector takes advantage of the morphological characteristics of retinal blood vessels to produce filter masks for emphasizing vessels. It works on the inverted green channel of original RGB images, where the vessels appear brighter than the background. First, a square patch of size $W \times W$ area is centered at each pixel position in the retinal image. Then, the average pixel intensity, termed $I_{a v g}^{W}$, is computed. To cater for vessels with different orientations, twelve lines of length $W$ pixels oriented at 12 different directions (angular resolution of $15^{\circ}$ ) passing through the centered pixels are laid out, and the average intensity along each line is computed. The line providing the highest average among all twelve lines is designated the 'winning line', and its value is defined as $I_{\max }^{W}$. The line response at the centered pixel is then computed as:

$$
R_{W}=I_{\max }^{W}(x, y)-I_{a v g}^{W}
$$

Where, care should be taken to pick the window size $W$ large should be chosen to ensure that the window is able to engulf the largest width vessel entirely. The basic line detector tends to merge closer vessels, extends cross-over points, and it produces shadow in the vicinity of the high-contrast vessels. 


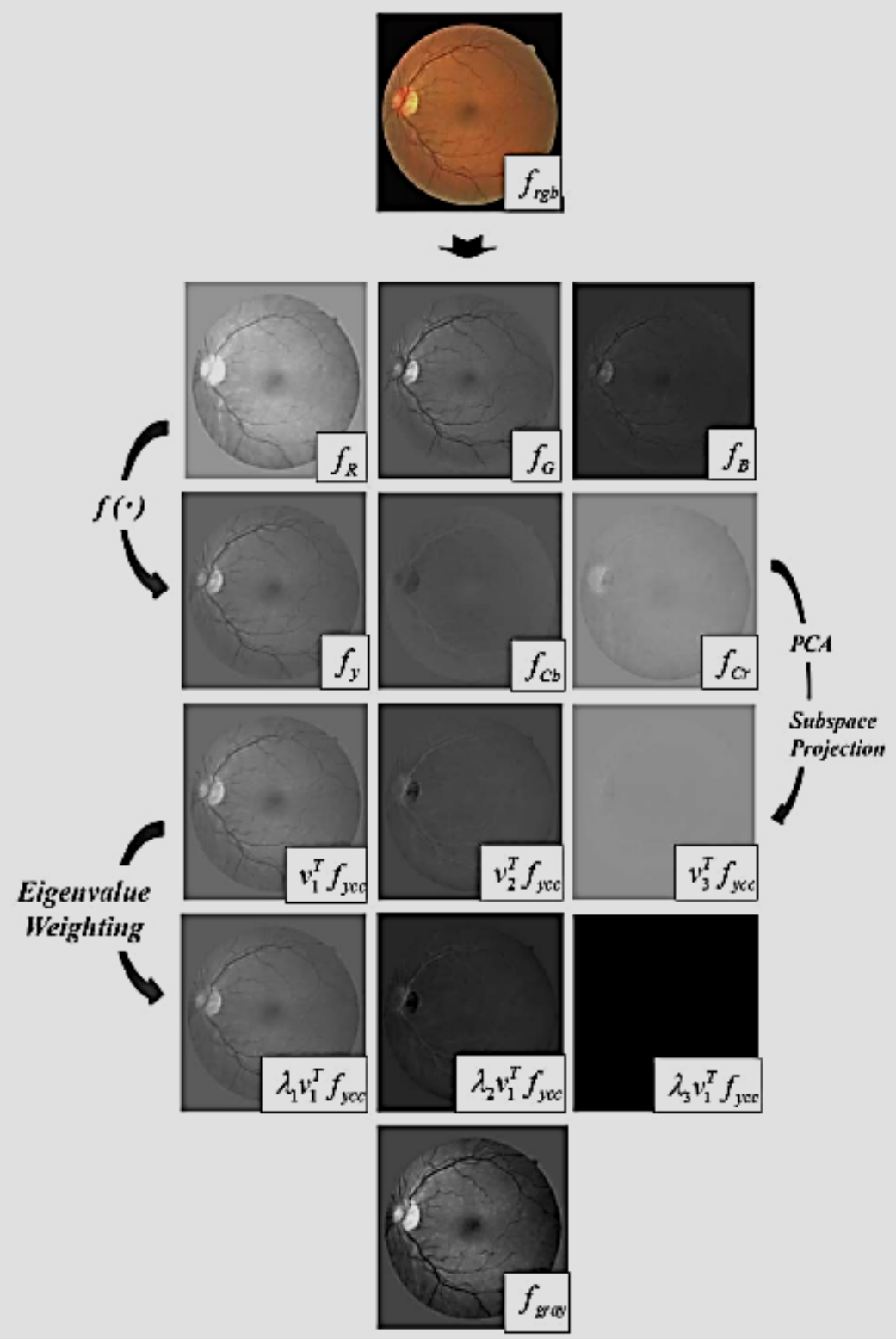



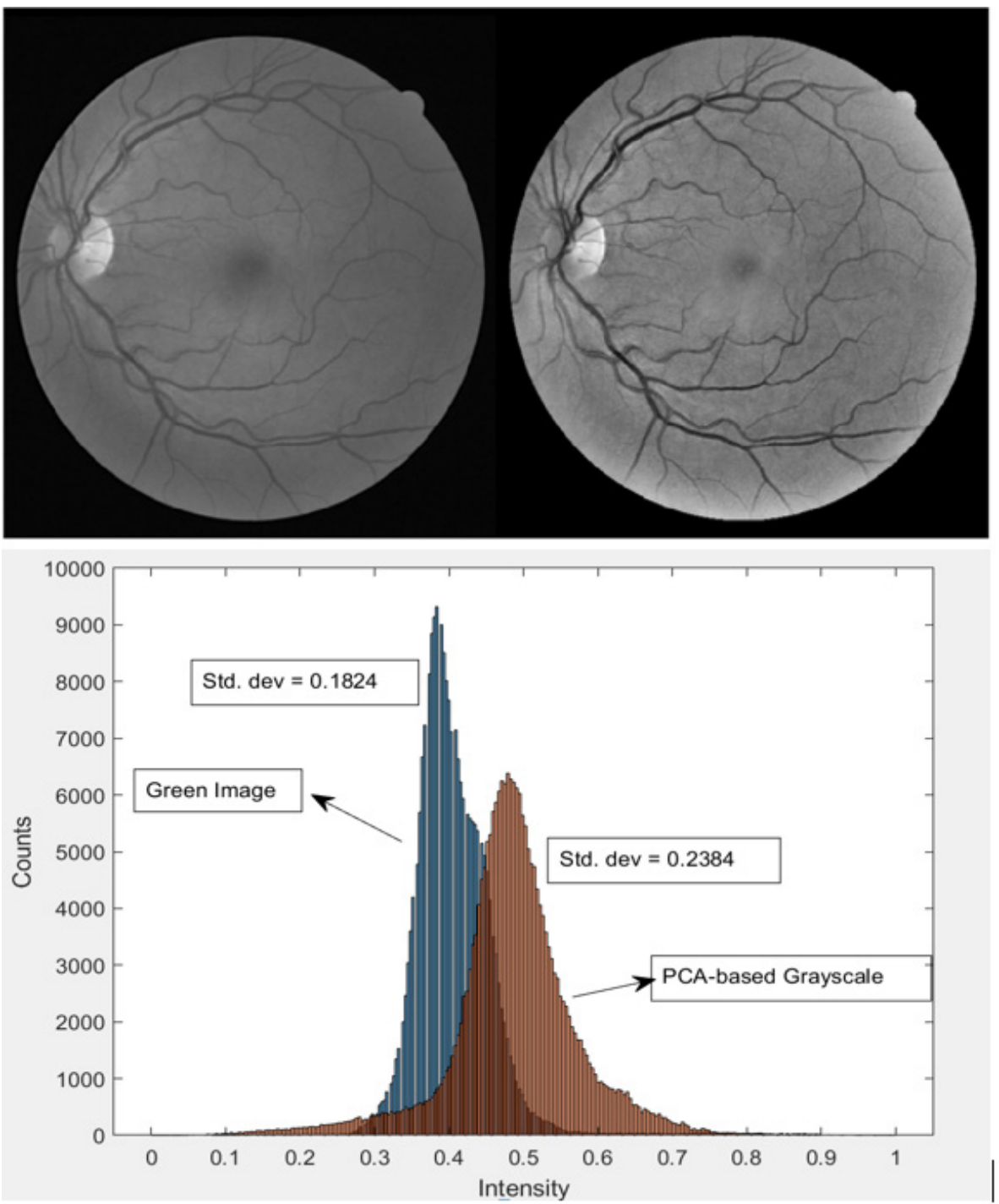

Figure 3. The Figure on the left shows the architecture for PCA-based color-togray conversion, while the figure on the top-right shows the green channel input as compared to PCA-based input. The figure on the bottom-right displays the histogram of both inputs, with PCA-based input having larger spread indicating better contrast as compared to green channel input.

To overcome the three drawbacks of the basic line detector mentioned above, Nguyen et al. [4] proposed a generalized multi-scale line detector, which uses a variable length of aligned lines in spatial scale. The generalized multi-scale line detector is defined as:

$$
R_{W}^{L}=I_{\max }^{L}(x, y)-I_{a v g}^{W}
$$

where $1 \leq L \leq W$ 
By providing a range of values for $L$, the multiscale line detector is developed. The main idea is that the shorter length line detector will avoid the inclusion of surrounding vessel pixels, and hence give correct false responses to closely located vessels, and at vessel crossovers, while the longer length line detector is helpful in detecting large diameter blood vessels. The output of the multiscale filtering process is a gray-scale image where vessels are made more prominent than other anatomical objects.

\subsection{ISODATA Threshold Operation}

The gray-scale image as output of the filtering process has to be converted to binary image for classification, where vessels pixels are depicted as white and rest of the background as black. For this to happen, we opted for Iso-data method, as envisioned in [10]. The method is iterative in nature at the end of which a suitable threshold is reached. Process starts with initial guess, and at each iteration a new threshold is generated in the following way: for a given a threshold $T_{i}$, the next threshold $T_{i+1}$ is the average of intensities above and below the previous threshold $T_{i}$. It is hoped that a better threshold $T_{i+1}$ emerges than that of $T_{i+1}$ for vesselbackground discrimination. The process terminates in a couple of iterations with the criterion of $T_{i+1}=T_{i}$. The success of the whole process is hinged upon the choice of the starting value of threshold $T_{0}$. In practice, the initial value, $T_{0}$, is smartly chosen by selecting a region in the image that is most likely to contain only pixels of the same class - background with help from appropriate information.

\section{RESULT AND DISCUSSION}

Retinal vessel segmentation method have been evaluated on two publically available data sets. Both DRIVE and STARE database have been widely used by researchers to test as their providers also made manual annotations available for performance evaluations.

One possible motivation to use PCA for color-to-gray scale conversion is related to the fact that if a 3D color gamut is more like an ellipsoid that is not aligned with any RGB axis, then PCA can help in finding the principal axis of the ellipsoid. In our case the color space $\mathrm{YCbCr}$ provides an elongated 3D object that have strong presence of a principal axis, moving along which provides us with vessel segmentation result, as depicted in Fig. 4. The PCA-based grayscale conversion is able to compute the first principal component that went along the major axis of the long skinny ellipsoid, whereas the second and third principal components are perpendicular to that and went out along the short circular cross section of the elongated object. The second and third principal components have much smaller significance and thus added to the final grayscale result with smaller weights. 


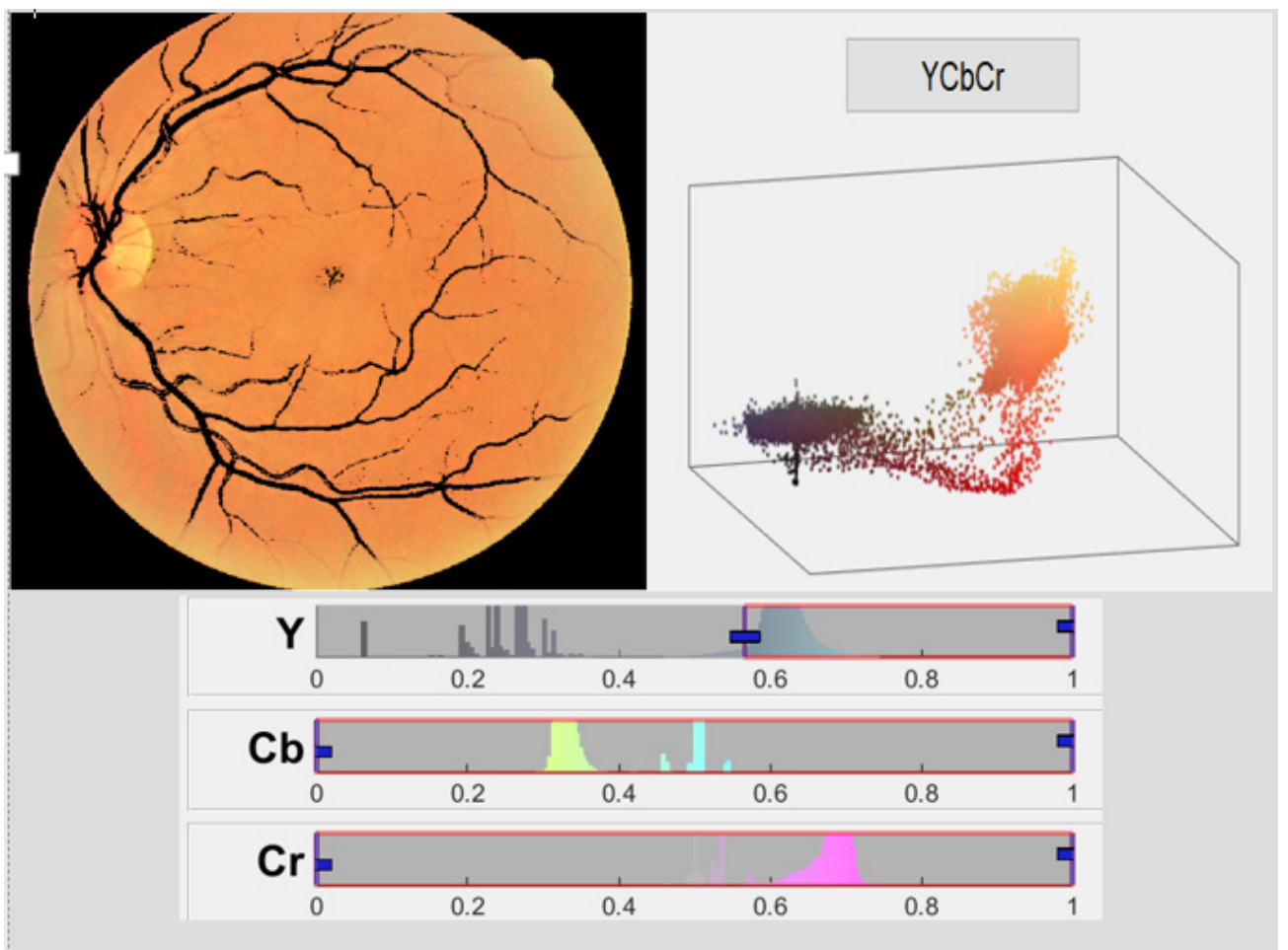

Figure 4: Conversion to Color Space YCbCr, and its 3D color gamut. The gamut clearly shows trend that majority of vessel pixels are aligned along $Y$ axis, making the $\mathrm{Y}$ channel more effective than $\mathrm{Cb}$ and $\mathrm{Cr}$ axis for vessel segmentation purposes. However, $\mathrm{Cb}$ and $\mathrm{Cr}$ channels can not be totally discarded as they do have contribution towards identifying small caliber vessels.

With reference to Fig. 5, we observe that in comparison to green channel image as input for Nguyen et al.'s multi-scale method [4], PCA-based grayscale input provides more details of the retinal vessels for the multiscale filtering method, even in the area of the optic disk and pathological lesions, which are considered challenging for vessel detection task. 


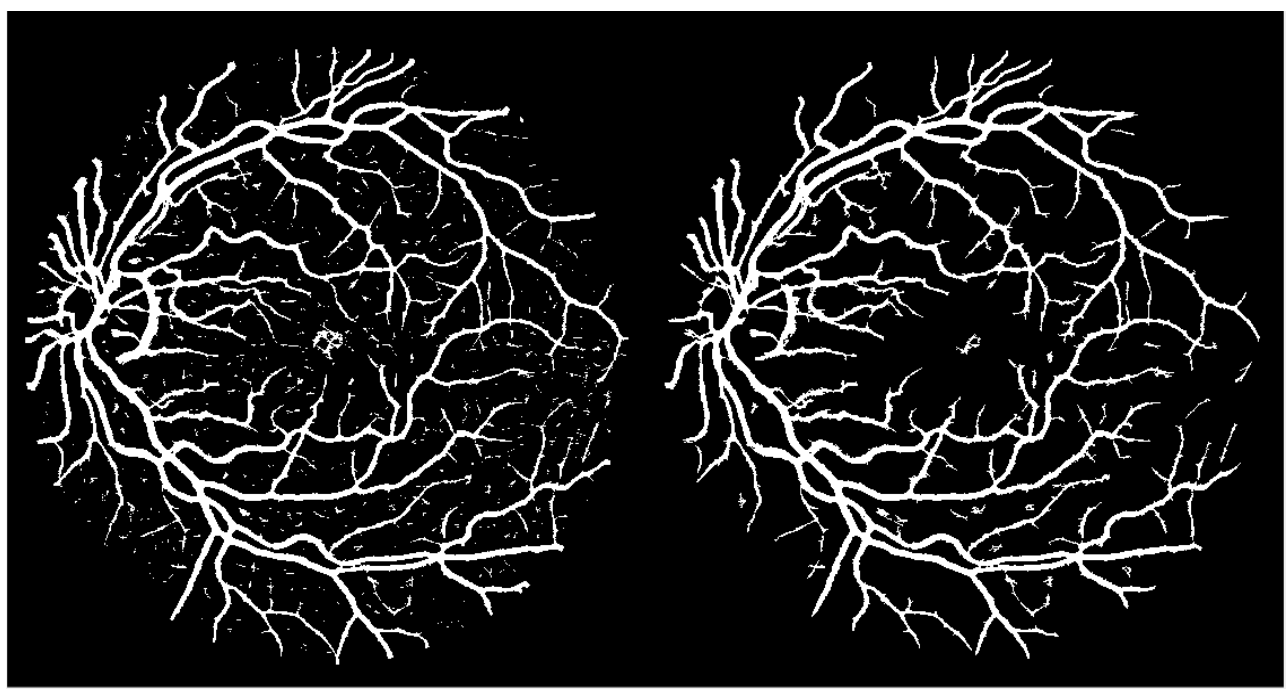

Figure 5. Left: The segmentation result due to Green channel image as input. Right: The segmentation result due to PCA-based Grayscale Image. The PCAbased grayscale provides an increased immunity to the background noise in favor of improved vessel tree extraction.

Table 1: DRIVE and STARE database

\begin{tabular}{lllll}
\hline Database & & Sensitivity & Specificity & Accuracy \\
\hline DRIVE & Green Input & 0.7322 & 0.9659 & 0.9407 \\
& PCA Input & 0.7364 & 0.9690 & 0.9417 \\
STARE & Green Input & 0.7317 & 0.9613 & 0.9324 \\
& PCA Input & 0.7350 & 0.9654 & 0.9338 \\
\hline
\end{tabular}

\section{CONCLUSION}

The impact of a discriminative gray-scale input made available to the multi-scale line filtering method for the purpose of vessel segmentaion has been analyzed. A certain choice of colorspace and then the use of weighted PCA was found more helpful in emphasizing vessels while suppressing background noise with a subsequent effect on the overall accuracy of automated vessel segmentaion process. The demonstrated effectiveness and robustness, together with its simplicity, make the PCA-based grayscale representation a suitable add-on to be integrated into a computer-assisted diagnostic tool for ophthalmic disorders.

\section{ACKNOWLEDGEMENT}

The authors would like to thank Effat University in Jeddah, Saudi Arabia, for funding the research reported in this paper through the Research and Consultancy Institute. 


\section{REFERENCES}

[1] Soares, J.V., Leandro, J.J., Cesar, R.M., Jelinek, H.F., Cree, M.J., Retinal vessel Segmentation using the 2-D Gabor wavelet and supervised classification, IEEE Transactions on medical Imaging, 2006, 25(9), 1214-1222

[2] Zhao, Y.Q., Wang, X.H., Wang, X.F., Shih, F.Y., Retinal vessels segmentation based on level set and region growing. Journal of Pattern Recognition, 2014, 47(7), 2437-2446

[3] Fraz, M.M., Remagnino, P., Hoppe, A., Uyyanonvara, B., Rudnicka, A.R., Owen, C.G., Barman, S.A., Blood vessel segmentation methodologies in retinal images - a Survey, Computer methods and programs in biomedicine, 2012, 108(1), 407-433

[4] U. T. Nguyen, A. Bhuiyan, L. A Park, and K. Ramamohanarao, An effective retinal blood vessel segmentation method using multi-scale line detection, Pattern Recognition, 2013, 46(3), 303-315

[5] M. Grundland and N. A. Dodgson, Decolorize: Fast, contrast enhancing, color to grayscale conversion, Pattern Recognition, 2007, 40(11), 2891-2896

[6] A. A. Gooch, S. C. Olsen, J. Tumblin, and B. Gooch, Color2gray: salience-preserving color removal, ACM Trans. Graph., 2005, 24(3), 634-639

[7] K. Smith, P.-E. Landes, J. Thollot, and K. Myszkowski, Apparent greyscale: A simple and fast conversion to perceptually accurate images and video, Computer Graphics Forum, 2008, 27(2), 193-200

[8] Ja-Won Seo; Seong Dae Kim, Novel PCA-based color-to-gray image conversion, 20th IEEE International Conf. on Image Processing (ICIP), 2013, 2279-2283

[9] Ardizzone, E., Pirrone, R., Gambino, O., \& Vitabile, S., Illumination correction on biomedical images, Computing and Informatics, 2014, 33(1), 175-196

[10] A. El-Zaart, Images thresholding using ISODATA techniques with gamma distribution, Pattern Recognition and Image Analysis, 2010, 20(1), 29-41 
